\title{
IR thermography investigation on induced transition by isolated roughness elements in high-speed flows
}

\author{
by F. Avallone*, F.F.J. Schrijer** and G. Cardone*
}

\begin{abstract}
*Dept. of Industrial Engineering, University of Naples, Via Claudio 21, 80125,Naples, Italy, francesco.avallone@unina.it **Delft University of Technology, Kluyverweg 1, 2629HS Delft, The Netherlands, f.f.j.schrijer@tudelft.nl

*Dept. of Industrial Engineering, University of Naples, Via Claudio 21, 80125,Naples, Italy, gcardone@unina.it
\end{abstract}

\section{Abstract}

Boundary layer transition in high-speed flows is a phenomenon that despite extensive research over the years is still extremely hard to predict. The presence of protrusions or gaps can lead to an accelerated laminar-to-turbulent transition enhancing the thermal loads and the skin friction coefficient. In the current investigation, high-resolution heat transfer measurements using infrared thermography are performed on the flow past several different roughness geometries (cylinders and pizza-box) at free stream Mach number equal to 7.5 and three unit Reynolds number (14. $\left.10^{6} \mathrm{~m}^{-1}, 11 \cdot 10^{6} \mathrm{~m}^{-1}, 8 \cdot 10^{6} \mathrm{~m}^{-1}\right)$. The roughness elements are applied on a plate that is oriented at a $5^{\circ}$ angle of attack with respect to the flow direction. For each Reynolds number the roughness element is positioned at $30 \mathrm{~mm}$ and at 60 $\mathrm{mm}$ from the leading edge. The measurements establish the roughness effectiveness in promoting transition and they provide insight into the flow topology.

\section{Introduction}

Boundary layer transition from laminar to turbulent flow is still an unpredictable phenomenon at high-speed. The transition of the boundary layer can be induced by several factors such as: amplification of the free stream disturbances, surface roughness or adverse pressure gradients. In this paper, 3D roughness elements placed in an incoming laminar boundary layer are experimentally investigated. The presence of such elements, that can appear in form of steps, joints or patterns left on a metal surface by ablation of the thermal protection system (TPS), can accelerate the laminarturbulent transition process enhancing the thermal loads and the skin friction coefficient [1]. The thermal loads are mostly due to the convective heat transfer, which depends on several parameters: re-entry trajectory, vehicle configuration and flow conditions [2]. In presence of a turbulent boundary layer the convective heat transfer rate can be 5 times higher respect to the laminar flows [3]. Therefore, the prediction of the laminar turbulent transition plays an important role for the vehicle's design and optimization [4]. On the other hand, in scramjet applications, artificial roughness elements are often employed for intentional tripping of the boundary layer on the forebody of the vehicle in order to prevent engine unstart and to minimize the flow non-uniformities at the entrance of the combustion inlet [5].

The mainly effect of the roughness is to move transition forward respect the smooth wall transition location. Van Driest and McCauley [6] defined a roughness element critical when it starts effecting on the transition location while they named effective a roughness able to move transition as close as possible to it. Although the definition of critical and effective roughness is rather clear, the identification of the onset of the transition location is quite ambiguous [1]. Moreover, the dependence of the laminar-turbulent transition by several parameters, such as Reynolds number and Mach number [6], size and geometry of the roughness, surface polishing, surface temperature and wind tunnel noise [7], make the comprehension of the physical mechanisms that lead to transition [8] very hard. Therefore, these parameters are often not well measured and do not exist any ground facility able to reproduce completely the flight environments: high Mach number, high Reynolds number, low noise and high enthalpy. Even if a general mechanism is not recognized, Schneider [1] reported three ways by which roughness can effect transition. In the first scenario the roughness generates a wake with streamwise vorticity and a possibly unstable shear layer. This wake may be unstable and may be dominated by the instability of the shear layer or of the trailing vorticity. The second way involves the growing of streamwise vorticity behind small roughness elements via instability mechanism such as stationary crossflow, Gortler or transient growth that can lead to instability. The third one is based on the coupling between the disturbances generate by the roughness element and the free stream disturbances that can lead to transition via the receptivity process.

Laminar to turbulent prediction is typically performed by means of empirical correlation obtained by systematic experimental campaign in ground facilities and then extrapolated to the few in-flight data [4]. A systematic work was carried out in support of the Hyper-X program [9] and in the frame of the ESA EXPERT program [10, 11]. Most of the proposed correlations lack of some physic aspects that lead to transition. Reda [12] suggested that the correlation could be obtained by comparing boundary layer properties at the roughness location with the characteristic lengths of the protrusion. Berry et al [13] proposed a simple correlation (Shuttle Roughness Criteria) based on boundary layer edge parameters: $\operatorname{Re}_{\theta} / M_{e}=C(h / \delta)^{-1}$. Although the constant parameter $\mathrm{C}$ is strictly dependent on the wind tunnel [1], Berry et al [14] showed good correlation with $C=200$ for critical or incipient roughness and $C=310$ for effective roughness. The correlation was obtained considering data from three separate models but in the same wind tunnel at the same Mach number and temperature ratio. Many other correlations have been proposed over the years, which use a higher number 
of parameters finding its field of applicability [15 - 17]. The necessity to predict transition with correlation that are widely applicable drives researches to better understand the fluid dynamic mechanism that are dominant in the roughness induced transition. Then, as much as possible experimental data are necessary.

Ground experiments are mainly performed by means of Pitot probes and hot wires but they are invasive techniques that may influence the fluid-dynamic features of the flow. The application of non-invasive technique, e.g. laser based and image based technique, is a necessity. Recently, in the Mach 10 wind tunnel at NASA Langley was applied the Nitrix oxide (NO) planar-laser induced fluorescence to investigate the hypersonic flow over a flat plate with and without a $2 \mathrm{~mm}$ radius hemispherical trip [18] and with a cylindrical roughness [19] showing interesting instantaneous features. The improvement of the experimental technique coupled with the availability of greater computational resources have enabled the use of direct numerical simulation (DNS) to study transition [20]. DNS results permits to better understand physical mechanics behind induced transition but few experimental data are available as benchmark. Recently, Iyev and Mahesh [21] performed DNS replicating the experiments carried out by Danehy et al [18]. In their paper they report a complete analysis of the wake in front and behind a hemispherical roughness elements with $h / \delta>1$ at three Mach number equal to $3.37,5.26$ and 8.23 . They observed that at the lowest Mach number the flow transition to turbulent exhibiting hairpin vortices in the wake and a 6-vortex system [22] in front of the roughness. Increasing the Mach number the flow is more stable a 4-vortex system is observed and the wake does not become turbulent. Moreover, they analyse the mean flow showing sensible difference in the wake features between the three cases. This paper is only the last of series of paper in which several configurations have been analysed in order to investigate several transitional scenarios. For example, Chang and Choudari [23] simulated the flow around a rectangular and cylindrical element at Mach number equal to 4.5 and 6.5 concluding that for these Mach numbers no self-sustaining vortex-generation was present and that convective instability might be the dominant. Groskpof et al [24] presented similar conclusion performing a bi-global stability analysis of the region behind a 3D pizza box shaped roughness.

In this paper IR thermography [25] is used to analyse the mean flow features upstream and downstream three roughness elements: two cylinders that differ from the diameter and a pizza-box shaped roughness. The height of the roughness elements is smaller than the boundary layer thickness at the roughness location. In the following first the description of the experimental setup and of the applied data reduction is presented. Then, in section 4 the description of the mean flow topology is discussed. A brief summary in section 5 concludes the paper.

\section{Measurement Technique}

Infrared thermography is used to obtain the surface heat transfer distribution in the vicinity and downstream of the roughness elements. The heat flux distribution is computed from the measured surface temperature using the semiinfinite slab model coupled with a least square approach [26] by solving (eq. 1). The model adopts the Trust Region Reflective algorithm as optimization routine from which it is able to evaluate the convective heat transfer coefficients $(h)$, the adiabatic wall temperature $\left(T_{a w}\right)$ and a delay parameter $\left(t_{d}\right)$.

$$
\begin{gathered}
k \nabla^{2}(T)=\rho c_{p} \frac{\partial T}{\partial t} \\
T(x, y, z, 0)=T_{w i} \\
\left.k \frac{\partial T(x, y, z, t)}{\partial n}\right|_{S}=q_{w}(t) \text { and } T(x, y, z \in F, t)=T_{F}(t) \\
q_{w}(t)=h\left(T_{w_{N U M}}-T_{a w}\right)+\sigma \varepsilon\left(T_{w_{N U M}}^{4}-T_{r}^{4}\right)
\end{gathered}
$$

The delay parameter $\left(t_{d}\right)$ is introduced such that start-up phenomena of the wind tunnel (that cannot be represented by the numerical model) can be taken into account. For example during the start-up transient the convective heat transfer coefficient and adiabatic wall temperature will vary strongly, while they are take to be constant in the model, which is a proper assumption when the tunnel is started. Therefore by introducing this delay only the temperature information is used for the period that the tunnel is properly started. As a matter of fact, Schrjier and Bannink [27] showed that stationary flow conditions start approximately $25 \mathrm{~ms}$ after the valve opening by measuring the pressure variation in the settling chamber. In figure 1 the comparison between the experimental temperature rise and the heat equation after the introduction of the delay is reported, as can be seen the first $30 \mathrm{~ms}$ are neglected.

The Stanton number $S t$ is obtained by non-dimensionalizing the wall heat flux using the free-stream parameters as shown in eq. 2 . The symbols $c_{p \infty}, \rho_{\infty}, T_{w}$ and $U_{\infty}$, respectively, represent the free stream constant pressure specific heat, the wall temperature and the free stream flow density and speed.

$$
S t=\frac{q_{w}}{\rho_{\infty} c_{p \infty} U_{\infty}\left(T_{w}-T_{a w}\right)}
$$

To visualize the heat flux distribution around and downstream of the roughness element the $\mathrm{K}$ contour [11], defined in eq. 3 , is used. $\mathrm{K}$ expresses the measured heat-flux normalized with the theoretical laminar and turbulent heat-flux predictions [28]. 


$$
K=100 \frac{q(x, y)-q_{\text {lam }}(y)}{q_{\text {turb }}(y)-q_{\text {lam }}(y)}
$$

For the interpretation the convention introduced by Tirtey et al [11] is adopted and is reported here for sake of clarity:

- $\quad K<30 \%$ : Laminar region;

- $\quad 30 \% \leq K \leq 70 \%$ : Transitional region;

- $\quad K \geq 70 \%$ : Turbulent region;

- $\quad K \geq 110 \%$ : Local structure overheating.

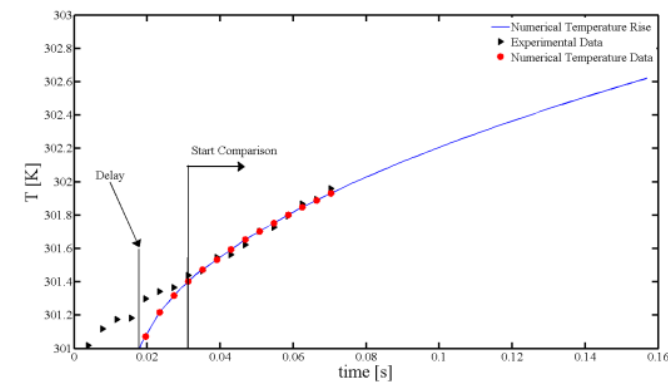

Figure 1. Example of the data reduction technique.

\section{Experimental Setup}

The experiments are performed in the Hypersonic Test Facility Delft [27]. The HTFD is a cold hypersonic wind tunnel that operates according to the Ludwieg tube concept that provides Mach 7.5 airflow in a $350 \mathrm{~mm}$ test section. The flow in the test section is optically accessible for imaging through three windows. Three nominal test conditions called low, medium and high Reynolds number have been investigated and, respectively, correspond to a free stream total pressure of 17, 22 and 28 bar. The unit Reynolds number is respectively equal to $8 \cdot 10^{6} \mathrm{~m}^{-1}, 11 \cdot 10^{6} \mathrm{~m}^{-1}$ and $14 \cdot 10^{6}$ with a total temperature of $579 \mathrm{~K}$. The typical run time is $0.1 \mathrm{~s}$. The free stream conditions are summarized in table 1 . According to Schrijer et al [29] the measurement repeatability associated to the operation of the Ludwieg tube facility is better than $1 \%$.

Table 1. Free stream flow properties.

\begin{tabular}{lccc}
\hline Parameter & & Quantity & \\
\hline$M_{\infty}$ & 7.5 & & \\
$U_{\infty}(\mathrm{m} / \mathrm{s})$ & 1000 & & \\
$T_{0}(\mathrm{~K})$ & 579 & 22 & 17 \\
$P_{0}($ bar $)$ & 28 & $11 \cdot 10^{6}$ & $8 \cdot 10^{6}$ \\
$R e_{\text {unit }}\left(\mathrm{m}^{-1}\right)$ & $14 \cdot 10^{6}$ & & \\
\hline
\end{tabular}

The IR themography measurements are performed using a CEDIP Titanium 530L IR system. The camera has a mercury cadmium telluride (MCT) quantum detector array of 320 by 256 pixels and a spectral response of 7.7-9.3 $\mu \mathrm{m}$. The camera has a maximum frame rate of $250 \mathrm{~Hz}$ at full resolution. The spatial resolution is $1.57 \mathrm{pixels} / \mathrm{mm}$. The optical access in the IR wavelength regime is provided by means of a Germanium window that has a transmissivity of approximately 0.8 . The camera was calibrated using a blackbody and the Germanium window was included in the calibration process to take into account the reduced transmissivity. The camera is setup under an angle with respect to the germanium window to prevent self-reflection. Furthermore, the camera is shielded from the environment using black sheets to prevent additional reflections from the background and window. A sketch of the camera set-up is given in Figure 2 (a). 


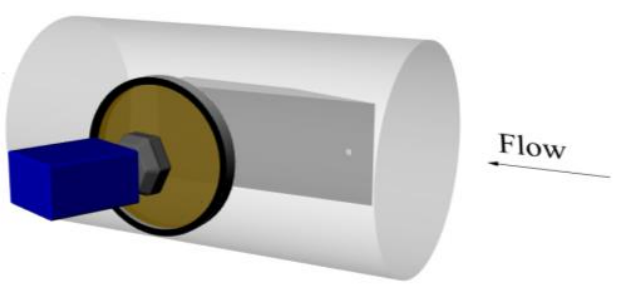

(a)

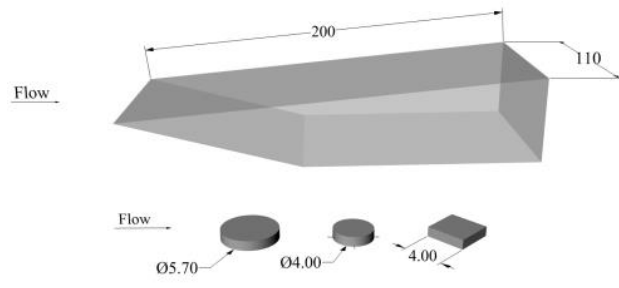

(b)

Figure 2. Sketch of the experimental setup (a) and wind tunnel model (b).

The wind tunnel model is a planar $5^{\circ}$ ramp, having a length of $200 \mathrm{~mm}$ and a width of $110 \mathrm{~mm}$ (see figure $2 \mathrm{~b}$ ). It is installed in the test section, mounted on a sting from its rear. The model is made out of Makrolon and the nose is accurately finished to avoid leading edge instabilities [30]. Makrolon is characterized by a conductivity equal to $k=$ $0.20 \mathrm{~W} / \mathrm{m} \mathrm{K}$ and surface emissivity of $\varepsilon=0.88$. In addition, this polycarbonate material can withstand temperatures up to $120^{\circ} \mathrm{C}$ without considerably changing the material properties. During the experimental campaign, the viewing angle with respect to the model surface was kept less than 50 degrees such that the emissivity could be regarded as independent of the viewing angle.

The initial model surface temperature is typically $300 \mathrm{~K} \pm 0.3 \mathrm{~K}$. For the high Reynolds test condition, a maximum wall temperature increase of $4 K$ is observed in the laminar flow region, while the temperature rise reaches $7 \mathrm{~K}$ in the turbulent wake. According to van Driest and Blumer [16] and Tirtey et al [11], this moderate wall temperature variation is not expected to have a significant effect on the following analysis.

In this study three roughness elements are investigated: two cylinders and a pizza-box like geometry. They are sketched in figure 2 (b). All of the elements have a height (h) of $1 \mathrm{~mm}$. The two cylinder shaped roughness elements have a diameter of $4 \mathrm{~mm}$ or $5.7 \mathrm{~mm}$ and they will be referred to as the large (cylL) or small (cylS) cylinder. The width of the pizza-box element is $4 \mathrm{~mm}$. The roughness elements are mounted at $x_{r}=30 \mathrm{~mm}$ and $x_{r}=60 \mathrm{~mm}$ from the leading edge along the symmetry axis. All the tests have been performed at zero pitch and yaw angles. The flat plate laminar boundary layer properties at both roughness locations are summarized in table 2.

Table 2. Boundary layer properties at roughness location.

\begin{tabular}{lcccccc}
\hline$R e_{\text {unit }}\left(m^{-1}\right)$ & \multicolumn{3}{c}{$14 \cdot 10^{6}$} & \multicolumn{2}{c}{$11 \cdot 10^{6}$} & \multicolumn{2}{c}{$8 \cdot 10^{6}$} \\
\hline$x_{r}(\mathrm{~mm})$ & 30 & 60 & 30 & 60 & 30 & 60 \\
$\delta_{99}(\mathrm{~mm})$ & 1.1 & 1.5 & 1.2 & 1.7 & 1.4 & 2 \\
$\theta(\mathrm{mm})$ & 0.13 & 0.18 & 0.15 & 0.21 & 0.17 & 0.24 \\
$R e_{\theta}$ & 1785 & 2450 & 1670 & 2307 & 1305 & 1830 \\
\hline
\end{tabular}

The ratio of roughness height to boundary layer thickness $(\delta)$ is reported in table 3 , the values for $h / \delta$ range from 0.5 to 0.9 . In order to predict whether the roughness elements are able to induce transition the Shuttle Roughness Criteria (SRC) $\operatorname{Re}_{\theta} / M_{e}=\left(C(h / \delta)^{-1}\right)$ is used and the results are displayed in figure 3 . In the criterion, $M_{e}$ is the Mach number at the edge of the boundary layer at the roughness location and the constant $C$ is equal or larger than 200 for critical roughness (transition downstream of the element) and equal or larger than 310 for effective roughness (transition at the location of the element, Berry et al [14]). In figure 3 filled symbols represents $x_{r}=30 \mathrm{~mm}$ while open symbols $x_{r}=60 \mathrm{~mm}$.

Table 3. $k / \delta$ ratio at $x_{r}=30 \mathrm{~mm}$ and $x_{r}=60 \mathrm{~mm}$ for the investigated roughness elements.

\begin{tabular}{ccccccc}
\hline & \multicolumn{3}{c}{$h / \delta @ x_{r}=30 \mathrm{~mm}$} & \multicolumn{3}{c}{$h / \delta @ x_{r}=60 \mathrm{~mm}$} \\
\hline $\operatorname{Re}_{\text {unit }}\left(\mathrm{m}^{-1}\right)$ & $8 \cdot 10^{6}$ & $11 \cdot 10^{6}$ & $14 \cdot 10^{6}$ & $8 \cdot 10^{6}$ & $11 \cdot 10^{6}$ & $14 \cdot 10^{6}$ \\
& 0.71 & 0.83 & 0.90 & 0.5 & 0.58 & 0.67 \\
\hline
\end{tabular}

Figure 3 clearly shows that the same roughness elements can be critical or effective depending on the free stream unit Reynolds number. Particularly, from the figure it can be seen that at low Reynolds number any elements is expected to trip the boundary layer while at the medium and high Reynolds number the roughness elements are expected to be critical. 


\section{Experimental Results}

The discussion of the experimental results is organized as follow: first the streamwise Stanton number along the centerline is presented. Then, the wake features are analyzed by means of the $\mathrm{K}$ contour in which also a discussion about the flow topology upstream and downstream the roughness elements is made.

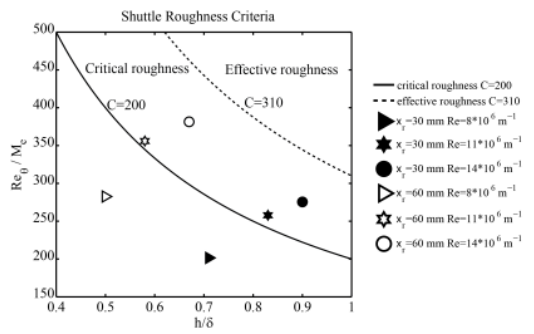

Figure 3. Shuttle roughness criteria: $C=200,310$ for critical and effective roughness respectively.

\subsection{Streamwise Stanton number distribution}

The streamwise Stanton number along the model centerline gives the immediate indication of the laminar or of the turbulent topology of the roughness wake. In figure 4 the $S t$ is plotted versus the non-dimensional streamwise coordinate for all the investigated roughness elements. The streamwise coordinate is non-dimensionalized with respect to the roughness location $\left(x_{r}\right)$. Figure 4(a) shows the results for the roughness placed at $x_{r}=30 \mathrm{~mm}$ while figure 4(b) depicts the results for $x_{r}=60 \mathrm{~mm}$. In these figures the theoretical laminar (dashed line) and turbulent (continuous line) Stanton number calculated with the reference temperature method [28] is plotted for the low $\left(8 \cdot 10^{6} \mathrm{~m}^{-1}\right)$, medium $\left(11 \cdot 10^{6} \mathrm{~m}^{-1}\right)$ and high $\left(14 \cdot 10^{6} \mathrm{~m}^{-1}\right)$ Reynolds number. For each configuration, the predicted laminar $S t$ is compared to the value that is measured outside of wake to verify that the incoming boundary layer is indeed laminar. For sake of clarity, in figure 4 (a) and (b) the out of wake St is reported for the three Reynolds numbers only for the pizza-box roughness element. As can be seen, the experimental $S t$ perfectly agrees with the predicted laminar values for all Reynolds numbers as.

From figure 4 it can also be clearly observed that the Stanton number in the wake of the roughness element increases with the Reynolds number. This can be explained by the fact that the boundary layer thickness $(\delta)$ at the roughness location becomes thinner and that $h / \delta$ increases and the roughness becomes more effective. When comparing figure 4(a) to 4(b), it is found that the Stanton number is lower in the latter. Following the same reasoning as before it can be concluded that the roughness perturbs the boundary layer more when it is positioned closer to the leading edge such that $h / \delta$ is larger. However comparing the various roughness elements, it is visible that the $S t$ depends on both the shape and the frontal area of the roughness for a given $h / \delta$. This concept is strongly highlighted in figure 4 (a) for the high Reynolds number. By comparing the pizza-box roughness and the large cylinder at the high Reynolds number, the $S t$ shows different trends downstream of the element. In the range $1.5<x / x_{r}<2.4$ the large cylinder is characterized by a St slightly lower that the predicted laminar value. This effect can be explained by the presence of a strong vortex pair downstream of the element that creates an upwash and therefore decreases the local heat transfer. For the pizza-box element a higher $S t$ is measured. In the range $2.4<x / x_{r}<3.4$ the Stanton numbers are comparable while for larger values of $x / x_{r}$ the patterns are rather different. For both the elements the $S t$ slope changes indicating the start of the transitional boundary layer that becomes turbulent where the Stanton number reaches its peak value. The transition to turbulent shows a different trend depending on the geometry: the large cylinder is characterized by a larger slope and a higher peak when compared to the pizza-box element moreover it exhibits a higher Stanton number with respect to the turbulent reference value. These observations confirm that the shape is a parameter that strongly influences the transition location and the overheating. For the small cylinder in the region directly downstream of the element $\left(1.5<x / x_{r}<2\right)$ the Stanton number is higher compared to large cylinder and pizza-box elements. This suggests that the vortical structures generated at the roughness edge are positioned closer to the centreline and therefore the local heat transfer is increased at this location. Furthermore, the transition to turbulence is less pronounced and the heat transfer pattern, represented by the s-shaped curve, has the same shape compared to the large cylinder one but it is scaled down reaching a lower peak value. Then, both the shape and the frontal area influence the transition to turbulence: the shape influences the transitional pattern while the frontal area influences the transition location and the maximum value of $S t$. Similar observations can be made for the medium Reynolds number where the same characteristics can be observed for the different roughness elements. The medium Reynolds number case differs from the high Reynolds number case only for the maximum St. For the low Reynolds number and $x_{r}=30 \mathrm{~mm}$ the Stanton number decreases almost monotonically when going downstream revealing that transition does not happen and the boundary layer remains laminar. However, the Stanton number is higher than the predicted laminar one due to the presence of the streamwise vortices that influence the heat transfer at the centreline. A similar behaviour can be observed for $x_{r}=60 \mathrm{~mm}$ (figure $4 \mathrm{~b}$ ). Particularly, for the low Reynolds number transition does not start and the Stanton number decreases going downstream while for the medium Reynolds number a weak increase can be detected. It suggests that the boundary layer is transitional but it is still far away from becoming fully turbulent. 
The above results agree well with the predictions obtained using the Shuttle Roughness Criterion. At low Reynolds number the roughness elements do not trip the boundary layer while at the high Reynolds number transition occurs somewhere downstream of the element so that they can be categorized as being critical. It is also interesting to highlight that the SCR predicts that the roughness is weakly effective for the medium Reynolds number when they are positioned at $x_{r}=60 \mathrm{~mm}$. Indeed, for these conditions only a weak increase of the Stanton number is measured.

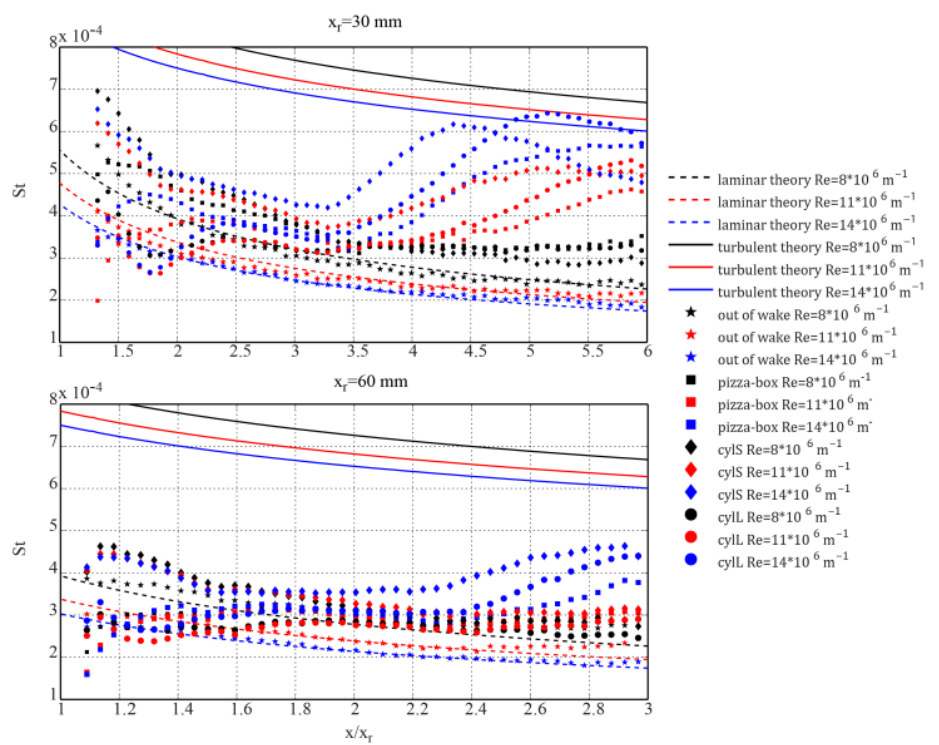

Figure 4. Streamwise Stanton number: (a) $x_{r}=30 \mathrm{~mm}$; (b) $x_{r}=60 \mathrm{~mm}$.

According to Schneider [1], it is possible to give a clear definition of transition location but it is difficult to detect the exact point in practice. In the following the transition location is defined as the point, along the streamwise location, at which the slope of the Stanton number at the centerline changes from negative to positive. The Reynolds number evaluated at the transition location is plotted versus the Reynolds number evaluated at the roughness location in figure 5 . From this figure it appears that the transitional Reynolds depends almost linearly on the Reynolds number evaluated at the roughness location. The slope changes by changing the frontal area and the roughness geometry. This figure also shows that the large cylinder is the most effective in tripping the boundary layer. Generally, it confirms that the cylindrical shape is more effective in perturbing the boundary layer. Concluding this section, the effect of both the shape and the impact area are not separable at the centerline.

\subsection{Wake topology}

IR thermography allows measuring the 2D heat transfer map on the model surface permitting to better understand the boundary layer topology upstream and downstream the roughness elements. In figure 6 the K contour (as introduced in section 2 eq. 3) is displayed for all the roughness elements at the medium (left) and high Reynolds number (right). The low Reynolds number is not shown because for these conditions the roughness elements are unable to trip the boundary layer. From the top to the bottom the small cylinder (figures 6a-6d), the large cylinder (figures 6e-6h) and the pizza-box elements (figures 6i-6l) are plotted. In these figures the roughness elements are contoured in blue but it does not have any physical meaning. In the following the flow field around and downstream of the roughness element is analyzed starting from the upstream separation and moving downstream.

Directly upstream of the roughness element the laminar boundary layer separates and forms one or multiple horseshoe vortices. Various researchers at subsonic and supersonic speed [21, 22, 31] observed this phenomenon. For the flow around an hemispherical bump placed in an incoming laminar boundary layer at Mach number ranging from 3.37, 5.26 and 8.23 lyer and Mahesh [21] stated that upstream of the roughness the vortex system can be formed by 4 or 6 vortices depending on the Reynolds number, the Mach number and $D / \delta^{*}$ ratio where $\delta^{*}$ is the displacement thickness at the roughness location. They found good agreement with the subsonic experiments carried out by Beker [22] for incompressible flow past a cylinder placed on a flat plate. These vortices transport higher-temperature fluids toward the wall and lower-temperature fluid away from the wall causing the increase in the heat flux (and thus also in $\mathrm{K}$ ) in the region between them. In the current experiments, the same features are detected. Indeed, in figure 6 (j) secondary streaks are clearly visible that pass in front of the element while in figure 6 (f) they are evidently not present. When these secondary structures are present a local $\mathrm{K}$ maximum is measured. By plotting the spanwise $\mathrm{K}$ just behind the roughness element, as reported in figure 7 , it is possible to extract information about the system of spanwise vortices that are formed upstream. In this figure the value of $\mathrm{K}$ as function of the spanwise coordinate is displayed at $x / x_{r}=1.3$ and $R e_{\text {unit }}=14 \cdot 10^{6} \mathrm{~m}^{-1}$ for the 3 roughness elements. As stated before a local maximum in the heat transfer is present in the region between two vortices. So, for the large and small cylinder elements two peaks are found which indicates that 
there are 4 vortices present ( 2 at either side of the centerline). Then, for the pizza-box element an additional and considerably smaller peak is found at approximately $y / h=6$ and $y / h=-6$, which hints at the presence of a 6-vortex system. In figure 7 it can be seen that it is more evident for the pizza-box respect to the large cylinder. The latter shows a region of almost constant $\mathrm{K}$ suggesting the presence of weak flow structures. Instead, the small cylinder does not show any local maximum suggesting the absence of secondary vortices. In figure 8 the number of upstream spanwise vortices is plotted as a function of $R e_{D} / M$ and $\left(D / \delta^{*}\right)(h / \delta)$. In the same figure the data reported by lyer and Mahesh [22] is plotted with labeled black squares. From the analysis of the figure it can be argued that for approximately $\left(D / \delta^{*}\right)(h / \delta)<$ 2 a 4 -vortex system is detected while for higher $\left(D / \delta^{*}\right)(h / \delta)$ a 6 -vortex system is present. This 6 -vortex system becomes stronger and more evident (looking at the heat transfer footprint) by increasing $\left(D / \delta^{*}\right)(h / \delta)$ becoming also highly unsteady according to lyer and Mahesh [22].

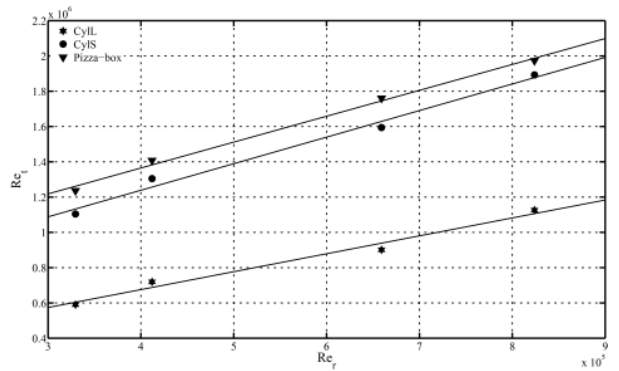

Figure 5. Reynolds number evaluated at the estimated transition $\left(R e_{t}\right)$ location versus the Reynolds number evaluated at the roughness location $\left(R e_{r}\right)$.

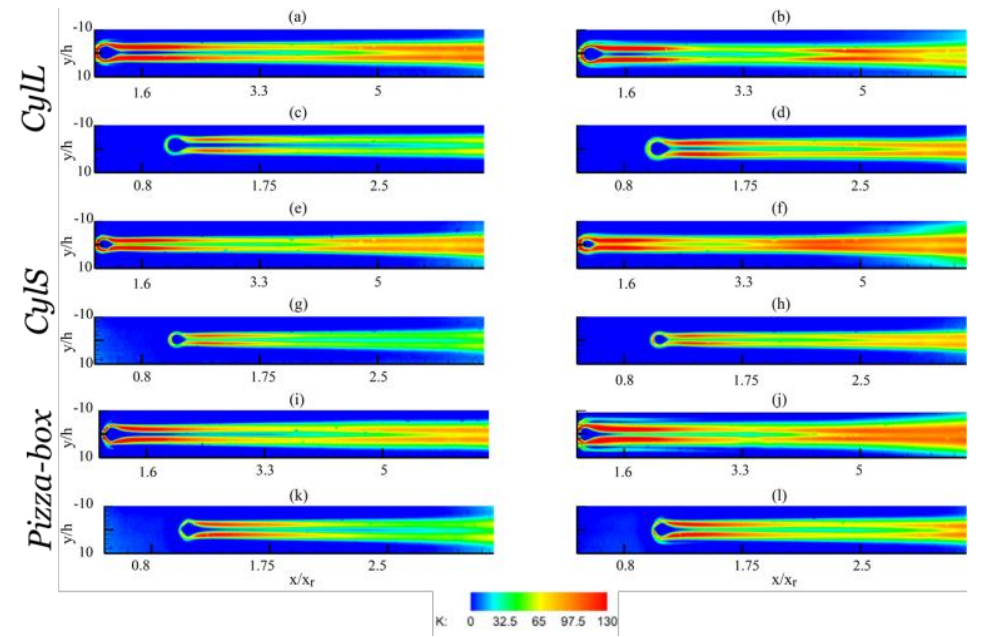

Figure 6. $K$ contour for the investigated roughness elements at Re unit $=11 \cdot 10^{6} \mathrm{~m}^{-1}$ (left) and $R e_{\text {unit }}=14 \cdot 10^{6} \mathrm{~m}^{-1}$ (right).

Downstream of the roughness element, the horseshoe vortices continue as streamwise vortices. From figure 6 it is clear that just behind the roughness element there is a localized region or streak of high heat transfer characterized by large values of $\mathrm{K}$. It is visible that when going further downstream, the streaks expand toward the symmetry plane and the $\mathrm{K}$ intensity decreases gradually. These streaks are connected to the symmetry plane (SP) and off-symmetry plane (OSP) vortices also detected by lyver and Mahesh [22] and Tirtey et al [11]. As a matter of fact, when moving in streamwise direction each SP vortex moves closer to the symmetry plane due to the induced velocity by the image vortex and the low-pressure region created behind the roughness. Therefore, one vortex from both sides of the roughness moves closer to the symmetry plane giving rise to a counter-rotating vortex pair [22]. At the same time, along the centerline a minimum $\mathrm{K}$ is measured due to the upwash by the SP vortices. Away from the symmetry plane, counter rotating vortex pair (or two pairs in case of a 6-vortex system upstream) is formed as continuation of the vertical tube formed upstream the roughness. Then, the SP and OSP vortices induce a downwash in between them. In this case, the effect of the mirror vortices is to move the vortices away from each other.

The strength of the vortices decreases with increasing distance from the roughness elements as clearly visible from the decrease in $\mathrm{K}$ in figure 6 . The reduction in the heat transfer rate is mainly associated to the weaker SP vortices that are more susceptible to breaking down according to the experimental evidence of Avallone et al [32] and discussion of lyver and Mahesh [22]. 


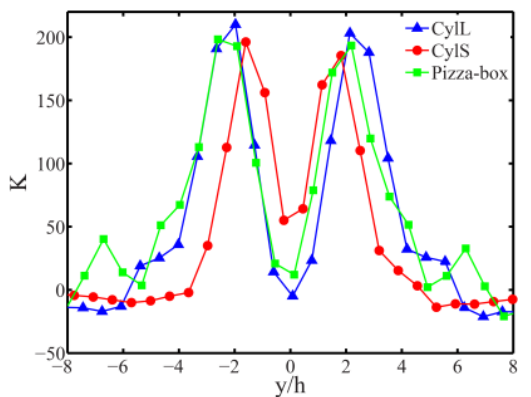

Figure 7. $K$ profile at $x / x_{r}=1.3$ and $R e_{\text {unit }}=14 \cdot 10^{6} \mathrm{~m}^{-1}$ for the three roughness elements.

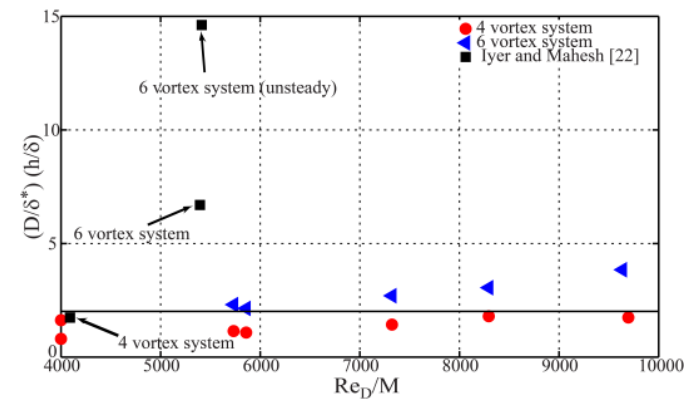

Figure 8. Number of vortices upstream of the roughness elements: the red circle represents 4-vortex system while the blue triangle the 6-vortex system. The black square symbol represents data obtained by the numerical simulation performed by lyer and Mahesh [22].

From a certain location, depending on the roughness geometry, position and unit Reynolds number, the flow undergoes transition as discussed in the previous section. The onset of transition is also visible by looking at the footprints of the vortical structures. Indeed, the intensity of the streaks becomes stronger until the boundary layer becomes fully turbulent. Even, in the fully turbulent region the footprint of the vortices is still clear for all the investigated configurations. This suggests that some coherent vortical structures are still present in the flow. The previous observations coupled with the experimental investigation using Tomographic PIV reported in Avallone et al [32] suggest that the OSP vortices still survive in the transitional region. Starting from the transition location and moving downstream the wake width increases. From figure 6 the turbulent spread of the wake ranges between $2^{\circ}$ and $2.5^{\circ}$ for all the investigated configurations. These data are in agreement with Fischer [33] who established that the lateral turbulent spreading angle at Mach number equal to 6.5 is less than $3^{\circ}$.

In the mean flow analysis an important aspect is represented by the maximum heat transfer rate measured downstream of the roughness elements with respect to the unperturbed one. From the spanwise $\mathrm{K}$ distribution, the amplitude $\mathrm{A}$ can be defined as one half of the maximum spanwise variation of $\mathrm{K}$ :

$$
A(x)=\frac{1}{2}\left(K_{\max }(x, y)-K_{\min }(x, y)\right)
$$

The result is plotted in figure 9 for the low (figure 9 a), medium (figure 9 b) and high (figure 9 c) Reynolds number. Starting from the low Reynolds number (figure 9 a) it is evident that the amplitude is similar for the larger cylinder and pizza-box element which have the same frontal area, while the amplitude is smaller for the small cylinder (having a smaller frontal area). When increasing the Reynolds number (figure 9b) a clear dependence on the frontal area can no longer be discerned. Furthermore when moving downstream the amplitude becomes similar for all elements.

When the flow undergoes the transition process an increase in Stanton number and thus also in $\mathrm{K}$ results. Since this increase in $\mathrm{K}$ is local (directly downstream of the element) also an increase in amplitude is registered. After the initial stages of flow development directly downstream of the element, the flow becomes fully turbulent and A becomes nearly constant and according to figure 9 the value will be equal to 50 . This effect is strongly present in figure 9 (c) where at high Reynolds number either the roughness positioned at $x_{r}=30 \mathrm{~mm}$ and $x_{r}=60 \mathrm{~mm}$ shows the turbulent plateau. Here, from approximately $x / x_{r}=2.7$ all the curves tends to the same amplitude value. Roughness elements positioned at $x_{r}=30 \mathrm{~mm}$ shows overheating regions characterized by higher $\mathrm{A}$ but after that they decreases to $\mathrm{A}$ values that are approximately equal to 50 . However, by plotting the streamwise amplitude it is possible to see that the maximum heat transfer is present just behind the roughness element and not in the wake even if it is higher than the turbulent expectations. On the other hand, increasing the Reynolds number the effect related to the shape and size of the elements becomes negligible when the interest is the measurement of the maximum heat transfer.

\section{Conclusions}


IR thermography is applied to investigate boundary layer transition induced by discrete roughness element placed on a flat plate in an incoming laminar boundary layer at asymptotic Mach number equal to 7.5. IR data have been analyzed by solving a 1D inverse heat transfer method based on a least square optimization in which also an time delay parameter is introduced to take into account the effects related to the unsteady startup of the wind tunnel. Three roughness elements (small and large cylinder, pizza-box) that differ from the shape and the size are investigated at three Reynolds number in order to perform measurements in a wide range of $h / \delta$ that goes from 0.5 to 0.9 . The measurements have highlighted that the large cylinder is more effective in tripping the boundary layer with respect to the small cylinder and the pizza-box element. From the footprints of the vortical structures just behind the roughness elements information about the system of spanwise vortices upstream the elements are obtained that are in good agreement with DNS data that are reported in literature. Particularly, for $\left(D / \delta^{*}\right)(h / \delta)>2$ a 6 -vortex system is detected instead of 4-vortex system. Lastly, by defining the streak amplitude, $A$, as one half of the maximum spanwise variation of $\mathrm{K}$ it has been observed that the transitional and turbulent regions are characterized by $A$ approximately equal to 50 .
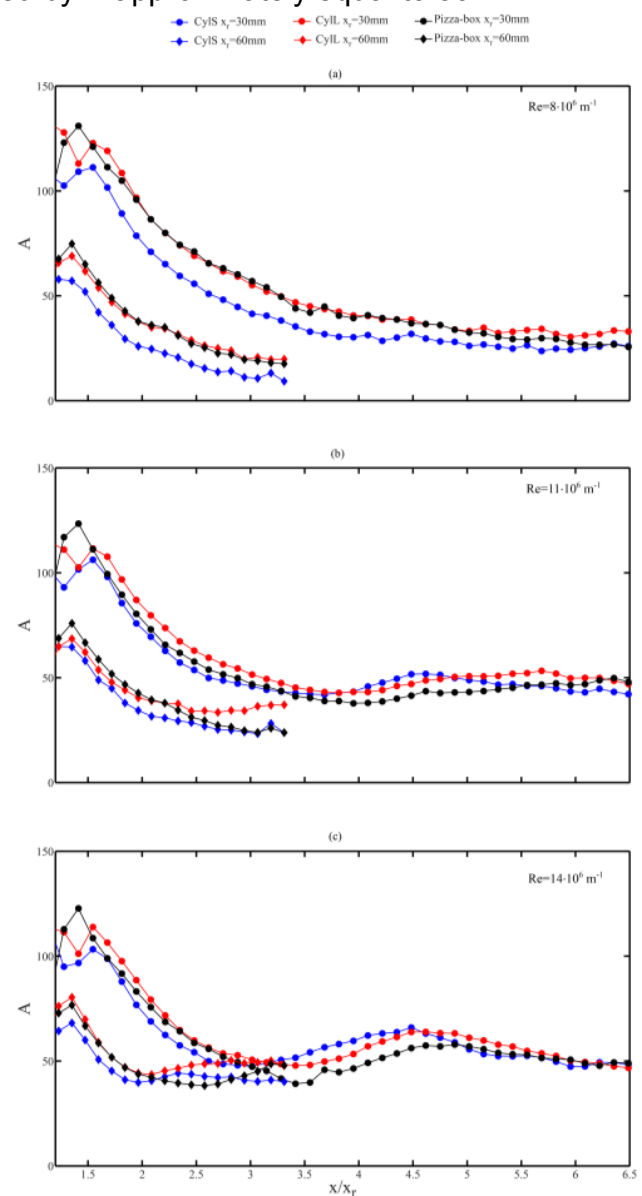

Figure 9. Streamwise amplitude evolution at $R e_{\text {unit }}=14 \cdot 10^{6} \mathrm{~m}^{-1}$ (a), Re $e_{\text {unit }}=11 \cdot 10^{6} \mathrm{~m}^{-1}$ (b) and $R e_{\text {unit }}=8 \cdot 10^{6} \mathrm{~m}^{-1}$

\section{REFERENCES}

[1] Schneider S.P, "Effects of Roughness on Hypersonic Boundary-Layer Transition". Journal of Spacecraft and Rockets, vol. 45(2), pp. 193-209, 2008.

[2] Schrijer F.F.J., Scarano F., van Oudheusden B.W., Bannink W.J., "Experiments on hypersonic roughness induced transition by means of infrared thermography". Proceedings of the Fifth European Symposium on Aerothermodynamics for Space Vehicles, paper ESA SP-563, Cologne (Germany), 2005.

[3] Anderson Jr. J.D., "Hypersonic and high temperature gas dynamics". McGraw-Hill, Boston (Massachusetts), 1989.

[4] Berry S.A., Horvart T.J., "Discrete Roughness Transition for Hypersonic Flight Vehicles". 45 ${ }^{\text {th }}$ AIAA Aerospace Sciences Meeting and Exhibit, paper AIAA 2007-0307, Reno (Nevada), 2007.

[5] Choudari M., Li F., We M., Chang C.L., Edwards J., Kegerise M., King R., "Laminar-Turbulent Transition behind Discrete Roughness Elements in a High-Speed Boundary Layer". $48^{\text {th }}$ AIAA Aerospace Sciences Meeting Including the New Horizons Forum and Aerospace Exposition, paper AIAA 2010-1575, Orlando (Florida), 2010.

[6] Van Driest E.R., McCauley W.D., "The effect of controlled three-dimensional roughness on boundary layer transition at supersonic speed". Journal of aeronautical sciences, vol. 27(4), pp. 261-271, 1960. 
[7] Schneider S.P., "Effects of High-Speed Tunnel Noise on Laminar Turbulent Transition". Journal of Spacecraft and Rockets, vol. 38(3), pp. 323-333, 2001.

[8] Stetson K.F., "Hypersonic Transition Testing in Wind Tunnels. Instability and Transition". Instability and Transition, vol.1, pp. 91-97, Springer-Verlag, Berlin, 1990.

[9] Berry S.A., Auslender A.H., Dilley A.D., Calleja J.F., "Hypersonic Boundary-Layer Trip Development for Hyper-X". Journal of Spacecraft and Rockets, vol. 38(6), pp. 853-864, 2001.

[10] Tirtey S.C., Chazot O., "Experimental investigation on hypersonic roughness induced transition for EXPERT program". 15 ${ }^{\text {th }}$ AIAA international space planes and hypersonic systems and technologies conference, paper AIAA 2008-2597, Dayton (Ohio), 2008.

[11] Tirtey S.C., Chazot O., Walpot L., "Characterization of hypersonic roughness-induced boundary-layer transition". Experiments in Fluids, vol. 50, pp. 407-418, 2011.

[12] Reda D.C, "Review and Synthesis of Roughness-Dominated Transition Correlations for Reentry Applications". Journal of Spacecraft and Rockets, vol. 39(2), pp. 161-167, 2002.

[13] Berry S.A., Bouslog S.A., Brauckmann G.J., Caram J.M., "Shuttle Orbiter Experimental Boundary-Layer Transition Results with Isolated Roughness". Journal of Spacecraft and Rockets, vol. 35(3), pp. 241-248, 1998.

[14] Berry S.A., Hamilton H.H., Wurster K.E., "Effect of computational method on discrete roughness correlations for shuttle orbiter". Journal of Spacecraft and Rockets, vol. 43(4), pp. 842-852, 2006.

[15] Potter J.L., Withfield J.D., "Effects of Slight Nose Bluntness and Roughness on Boundary Layer Transition in Supersonic Flows." Journal of Fluid Mechanics, vol. 12(4), pp. 501-535, 1962.

[16] Van Driest E.R., Blumer C.B., Wells C.S. Jr., "Boundary Layer Transition on Blunt Bodies-Effects of Roughness". AIAA Journal, vol. 5, pp. 1913-1915, 1967.

[17] Wool M.R., "Final Summary Report, Passive Nosetip Technology (PANT) Program". SAMSO, TR-75-250, 1975

[18] Danehy P.M., Bathel B., Ivey C., Inman J.A., Jones S.B., "NO PLIF study of hypersonic transition over a discrete hemispherical roughness elements". $47^{\text {th }}$ AIAA Aerospace Sciences Meeting Including The New Horizon Forum and Aerospace Exposition, paper AIAA 2009-394, Orlando (Florida), 2009.

[19] Danehy P.M., Ivey C.B., Inman J.A., Bathel S.B., McCrea A.C., Jiang N., Webster M., Lempert W., Miller J., Meyer T., "High-speed PLIF of hypersonic transition over discrete cylindrical roughness". $48^{\text {th }}$ AIAA Aerospace Sciences Meeting Including The New Horizon Forum and Aerospace Exposition, paper AIAA 2010-703, Orlando (Florida), 2010.

[20] Zhong X., Wang C., "Direct Numerical simulation on the receptivity, instability and transition of hypersonic boundary layer". Annual Review of Fluid Mechanics, vol. 44, pp. 527-561, 2012.

[21] lyer P.S., Mahesh K., "High-speed boundary-layer transition induced by a discrete roughness element". Journal of Fluid Mechanics, vol. 729, pp. 524-562, 2013.

[22] Baker C.J., "The laminar horseshoe vortex. Journal of Fluid Mechanics", vol. 95(2), pp. 347-367, 1979.

[23] Chang C.L., Choudari M.M., "Hypersonic viscous flow over large roughness elements". Theoretical and Computational Fluid Dynamics, vol. 25, pp. 85-104, 2011.

[24] Groskopf G., Kloker M.J., Marxen O., "Bi-global secondary stability theory for high-speed boundary layer flows". In Proceedings of the Summer Program, Center for Turbulence Research, 2008.

[25] Carlomagno G.M., Cardone G., "Infrared Thermography for convective heat transfer measurements". Experiments in Fluids, vol. 49(6), pp. 1187-1218, 2010.

[26] Avallone F., Greco C.S., Ekelschot D., "Image resection and heat transfer measurements by IR thermography in hypersonic flows", QIRT Journal, vol. 10 (2), pp. 188-206, 2013.

[27] Schijer F.F.J., Bannink W.J., "Description and flow assessment of the delft hypersonic Ludwieg tube". Journal of Spacecraft and Rockets, vol. 47, pp. 125-133, 2010.

[28] Eckert E., "Engineering relations for heat transfer and friction in high-velocity laminar and turbulent boundary-layer flow over surface with constant pressure and temperature". Transaction ASME, vol. 78, pp. 1273-1283, 1956.

[29] Schrijer F.F.J., Scarano F., van Oudheusden B.W., "Application of PIV in a Mach 7 double ramp flow". Experiments in Fluids, vol. 41, pp. 353-363, 2006.

[30] Le Duc A., Sesterhenn J., Friedrich R., "Instabilities in compressible attachment-line boundary layers". Physics of Fluids, vol. 18, pp. 044102, 2006.

[31] Simpson R.L., "Junction Flows". Annual Review of Fluid Mechanics, vol. 33, pp. 415-443, 2001.

[32] Avallone F., Ye Q., Schrijer F.F.J., Scarano F., Cardone G., "Tomographic PIV investigation of roughness induced transition in a hypersonic boundary layer". $17^{\text {th }}$ International Symposium on Applications of Laser Techniques to Fluid Mechanics, Lisbon (Portugal), 2014.

[33] Fischer M.C., "Spreading of a turbulent disturbance". AIAA Journal, vol. 10 (5), pp. 9757-9959, 1972. 